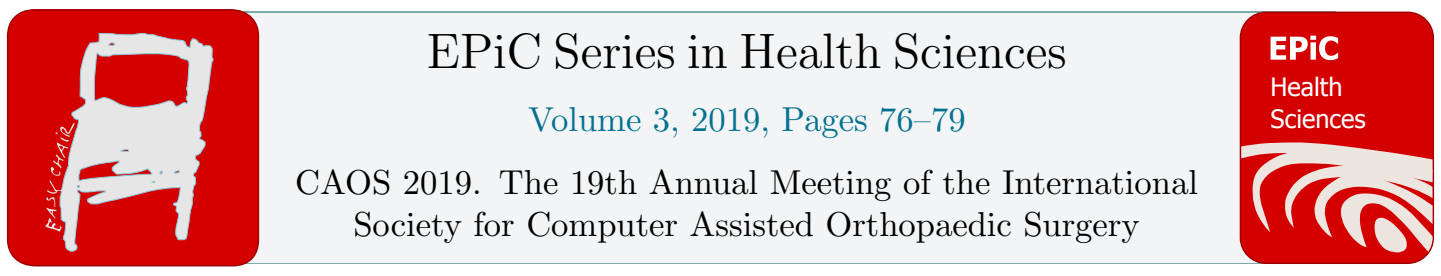

\title{
A 90-Day Episode-of-Care Cost Analysis of Robotic-Arm Assisted Total Knee Arthroplasty
}

\author{
Christina. Cool $^{1}$, David Jacofsky ${ }^{2}$, Kelly Seeger ${ }^{1}$, Andréa. Coppolecchia ${ }^{3}$; Nipun \\ Sodhi $^{4}$, Joseph O. Ehiorobo ${ }^{4}$, Michael A. Mont ${ }^{4}$
}

\author{
${ }^{1}$ Bakey Tilly; The Core Institute 2 ; ${ }^{3}$ Stryker Corporation; ${ }^{4}$ Lenox Hill Hospital, \\ Northwell Health; mmont@northwell.edu
}

\section{Introduction}

Although a number of non-surgical and surgical management modalities exist, total knee arthroplasty (TKA) has been considered the gold standard procedure for endstage knee $\mathrm{OA}^{1}$. For most patients, this surgery is highly successful, resulting in substantial improvements in quality of life and function ${ }^{2}$. However, despite the number of clinical and economic benefits of TKA, due to the high demand and volume of this procedure, the associated overall healthcare costs present substantial financial burden on payers. One way to potentially help contain healthcare costs is the utilization of technological advances, such as robotic-assistive technology, in the operating room $^{3}$. Therefore, the purpose of this study was to perform a cost analysis between roboticarm assisted TKA and manual TKA (mTKA) techniques. Specifically, we compared: 1) 90-day EOC costs, as well as several variables within the episode, including 2) index costs; 3) index lengths-of-stay (LOS); 4) discharge disposition; and 5) readmission rates.

\section{Methods}

A retrospective claims analysis was performed to provide longitudinal assessments of Medicare FFS beneficiaries who underwent rTKA and mTKA procedures performed between January 1, 2016 and March 31, 2017 (referred to as the index period), and included the following services: Inpatient, Outpatient, Skilled Nursing (SNF), and Home Health Agency (HHA). Patients were matched rTKA to mTKA in a 1-to-5 ratio. Patients were matched based on the following criteria: age, sex, race (as defined by 
CMS), geographic division (based on U.S. Census Bureau divisions), and high-cost comorbidities [including Chronic Obstructive Pulmonary Disease (COPD), Hypertension, Diabetes, Coronary Artery Disease (CAD), Smoking, Pneumonia, Cancer, and Obesity]. After PSM, 519 rTKA and 2,595 mTKA were identified and included for analysis. The overall 90-day EOC costs, including the index procedures, LOS, discharge dispositions, and readmissions were calculated. A Generalized Linear Model (GLM) was selected to test for statistical significance due to the data not being normally distributed. Statistical comparisons were made of EOC costs in the 90-day post-index period. A two-part model was created to conduct statistical analyses of the probability of having utilization as well as the associated costs in each post-operative cost setting. A binomial regression with log link was used to test the probability of having costs or utilization in the 90 -day post-index period in the first part of the model.

\section{Results}

Overall 90-day EOC costs for rTKA patients were found to be significantly less than that for mTKA patients $(\$ 18,568$ vs. $\$ 20,960)$. These amounts showed that rTKA patients have an $11 \%(\$ 2,391)$ lower average cost associated with their post-operative 90-day window of care $(\mathrm{p}<0.0001)$.

Overall index facility costs to the payer for rTKA patients were found to be significantly less than that for mTKA patients $(\$ 12,384$ vs. $\$ 13,024 ; p=0.0001)$. These amounts for rTKA patients demonstrates a 5\% (\$640) lower cost associated with their index facility costs.

Over $90 \%$ of patients in both cohorts utilized post-acute services, with rTKA accruing $\$ 1,744(25 \%)$ less costs than mTKA $(5,234$ vs. $\$ 6,978 ; p=<0.0001)$. Post-acute savings can be potentially attributed to rTKA patients being discharged to home with care (57 vs. $47 \%$; $\mathrm{p}<0.0001$ ) or self-care ( 28 vs. $24 \%$; $\mathrm{p}=0.0566)$ more frequently, and SNF less frequently (12.52 vs. $21.70 \%$; $<<0.0001)$.

In addition to discharge disposition, while over $90 \%$ of members in both cohorts utilized post-acute services, rTKA members utilized fewer post-acute services $(91 \%$ vs. $94 \% ; p=0.0654)$. Differences in utilization were most notable in the SNF and ER settings. Robotic TKA members were $47 \%$ less likely to have a SNF admission, and $16 \%$ less likely to utilize ER services in the 90 -day post-index period (14\% vs. $26 \%$; $\mathrm{p}<0.0001$ and $11 \%$ vs. $13 \%$; $\mathrm{p}=0.1726$,).

Among those members utilizing post-acute services, rTKA members experienced decreased post-index LOS and fewer visits. Most notably, rTKA members utilized fewer days in inpatient ( 4 vs. $7 ; p<0.0001)$ and SNF care (15 vs. 16; $p=0.0642$ ). Aside from inpatient services, rTKA members also utilized fewer outpatient and HHA visits, with rTKA members having 17\% fewer outpatient visits (3.00 vs. 3.59; $\mathrm{p}=0.0004)$, $13 \%$ fewer ER visits $(1.09$ vs. $1.25 ; \mathrm{p}=0.3545)$ and $16 \%$ fewer HHA visits compared to mTKA (11.94 vs. 14.28; $\mathrm{p}<0.0001)$. 
The aforementioned reductions in healthcare utilization resulted in decreased costs for the rTKA cohort. Members in the rTKA cohort averaged \$1,744 (25\%) fewer costs overall than those in the mTKA cohort $(\$ 5,234$ vs. $\$ 6,978 ; \mathrm{p}=0.0001)$. In addition to significant savings in the outpatient ( $\$ 699$ vs. $\$ 839 ; p=0.0019)$, SNF ( $\$ 7,201$ vs. $\$ 7,947 ; p=0.0230)$, and HHA settings $(\$ 3,045$ vs. $\$ 3,536 ; p<0.0001)$, savings were also realized in the inpatient and ER settings. rTKA members were also less likely to have costs for SNF admissions and ER visits as compared to mTKA (13.49\% vs. $25.55 \%$; $p<0.0001$ and $11.18 \%$ vs. $13.26 \%$; $p=0.1802$, respectively).

rTKA patients had a 90-day readmission reduction of $33 \%(\mathrm{p}=0.0423)$, when compared to $\mathrm{m}$ TKA patients.

\section{Discussion}

The results from this study show rTKA to be associated with significantly lower 90day EOC costs. These lower rTKA patient costs are likely attributable to the significantly lower index costs, increased likelihood of being discharged to home, shorter LOS, and decreased readmission rates, when compared to mTKA patient costs. Additional contributing factors may include a number of RAA-associated clinical, radiographic, and patient outcome advantages ${ }^{4-10}$. Future studies should build on these findings, by performing hospital and surgeon-specific cost analyses. Additionally, these data entertain further discussions regarding health-care cost-containment with operating-room technology utilization. Based on this data, robotic-arm assisted surgery appears to be cost-effective and provide added value for patients undergoing total knee arthroplasty. As a result of these findings, robotic-arm assisted surgery can be an effective tool in managing existing value based care programs while also delivering value given its ability to promote efficiencies through the EOC journey. 


\section{References}

1. Deshpande BR, Katz JN, Solomon DH, Yelin EH, Hunter DJ, Messier SP, et al. The number of persons with symptomatic knee osteoarthritis in the United States: Impact of race/ethnicity, age, sex, and obesity. Arthritis Care Res (Hoboken) [Internet] 2016;68(12):1743-1750.

2. Kurtz S, Ong K, Lau E, Mowat F, Halpern M. Projections of primary and revision hip and knee arthroplasty in the United States from 2005 to 2030. J Bone Joint Surg Am United States, 2007;89(4):780-785.

3. Mont MA, Khlopas A, Chughtai M, Newman JM, Deren M, Sultan AA. Value proposition of robotic total knee arthroplasty: what can robotic technology deliver in 2018 and beyond? Expert Rev Med Devices England, 2018;1-12.

4. Sultan AA, Piuzzi N, Khlopas A, Chughtai M, Sodhi N, Mont MA. Utilization of robotic-arm assisted total knee arthroplasty for soft tissue protection. Expert Rev Med Devices England, 2017;1-3.

5. Urish KL, Conditt M, Roche M, Rubash HE. Robotic Total Knee Arthroplasty: Surgical Assistant for a Customized Normal Kinematic Knee. Orthopedics United States, 2016;39(5):e822-7.

6. Khlopas A, Chughtai M, Hampp EL, Scholl LY, Prieto M, Chang T-C, et al. Robotic-Arm Assisted Total Knee Arthroplasty Demonstrated Soft Tissue Protection. Surg Technol Int United States, 2017;30:441-446.

7. Sodhi N, Khlopas A, Piuzzi NS, Sultan AA, Marchand RC, Malkani AL, et al. Erratum to: The Learning Curve Associated with Robotic Total Knee Arthroplasty. J Knee Surg Germany, 2018;31(4):370.

8. Marchand RC, Sodhi N, Bhowmik-Stoker M, Scholl L, Condrey C, Khlopas A, et al. Does the Robotic Arm and Preoperative CT Planning Help with 3D Intraoperative Total Knee Arthroplasty Planning? J Knee Surg Germany, 2018;

9. Marchand RC, Sodhi N, Khlopas A, Sultan AA, Harwin SF, Malkani AL, et al. Patient Satisfaction Outcomes after Robotic Arm-Assisted Total Knee Arthroplasty: A Short-Term Evaluation. J Knee Surg 2017;

10. Kayani B, Konan S, Tahmassebi J, Pietrzak JRT, Haddad FS. Robotic-arm assisted total knee arthroplasty is associated with improved early functional recovery and reduced time to hospital discharge compared with conventional jig-based total knee arthroplasty. Bone Joint J England, 2018;100-B(7):930937. 